\title{
SEMI-LIGHTPATH APPROACH FOR BANDWIDTH GUARANTEED PROTECTION IN IP- OVER-WDM NETWORKS
}

\author{
R.Gangopadhyay ${ }^{1}$, G.Prati ${ }^{2}$, N.Rao ${ }^{3}$ \\ ${ }^{1,3}$ Department of Electronics and Electrical Communication Engineering \\ Indian Institute of Technology, Kharagpur, India. \\ Tel: $+913222-78028, F a x:+913222-55303$ \\ e-mail: ranjancece.iitkgp.ernet. in \\ ${ }^{2}$ Scuola Superiore Sant 'Anna, Piazza Martiri della Libertà 33, 56127 Pisa, Italy \\ Tel:+39-050-970719, Fax:+39-050-9711208 \\ e-mail: giancarlo.prati@cnit.it
}

\begin{abstract}
The paper presents efficient algorithms for routing and wavelength assignment for dynamic lightpath establishment as well as bandwidth guaranteed path protection against single link failure in a WDM mesh network. Several protection strategies for multi-wavelength optical network have been studied to indicate their relative efficiency in terms of network blocking probability performance and resource requirement. Protection results in integrated routing scenario are also presented.
\end{abstract}

\section{INTRODUCTION}

The current trend in research on optical networking is focused on various issues related to IP (Internet Protocol) over WDM (Wavelength Division Multiplexing) technology. These may include transmission aspects in the physical fiber layer, and switching, routing, control and management issues in the data link layer. One of the important functionalities of the control layer is to route IP traffic from a source node $(s)$ to the destination node $(d)$ utilizing a lightpath (same wavelength in all the links determining the path from $s$ to $d$ ) or a semilightpath (a chain of wavelengths assigned link by link from $s$ to $d$ ). In general, the semilightpath algorithm (SLP) achieves simultaneous routing and wavelength assignment (RWA) for dynamic lightpath provisioning in a WDM network with or without $\lambda$-conversion facility at nodes. In the past, this algorithm [1] has been used extensively for WDM network design but without adequate consideration for network survivability. 
In the present paper we have exploited the SLP algorithm for solving the RWA problem in a WDM mesh network for a single link failure case and simultaneously guaranteeing cent percent protection to the disrupted traffic. Various strategies of protection: dedicated, partial shared and complete information have been implemented. In addition to the wavelength routing scheme, various strategies of protection such as dedicated path protection and shared path protection in the integrated routing scenario have also been investigated.

\section{PATH PROTECTION STRATEGIES}

We have considered four path protection schemes in our work, namely, dedicated path protection, shared path protection, partial shared path protection with exact reservation and complete shared path protection We consider a network of $\mathrm{N}$ nodes and $\mathrm{m}$ links. The call setup request $\mathrm{k}$ is considered as a duplex $\left(s_{k}, d_{k}\right)$. For the request $k, s_{k}$ specifies the ingress node and $d_{k}$ specifies the egress node. For each request, both an active path and a corresponding backup path have to be set up. Since all calls are to be protected, both active and backup paths need to use link disjoint paths.

We consider the following definitions [2] that will be required for various protection strategies we have adopted in our present studies. Let

$A_{i j}$ denotes set of active paths that use link $(i, j)$

$\mathrm{B}_{i j}$ denotes set of back-up paths that use link $(i, j)$

$F_{i j}$ denotes total bandwidth reserved by active paths that use link ( $\left.i, j\right)$

$\mathrm{G}_{i j}$ denotes total bandwidth reserved by backup paths that use link $(i, j)$

and the residual bandwidth $\mathrm{R}_{\mathrm{ij}}=\mathrm{C}_{\mathrm{ij}}-\mathrm{F}_{\mathrm{ij}}-\mathrm{G}_{i j}$, where $\mathrm{C}_{\mathrm{ij}}$ is the capacity of link $(i, j)$.

\subsection{Dedicated protection}

In this case the only information known at the time of routing the current request is the residual bandwidth $R_{i j}$ for each link $(i, j)$ in the network. Since no information is known other than $\mathrm{R}_{\mathrm{ij}}$ we have no means of finding out which active paths can share the backup links. Hence, the complete resource required by the requested call has to be reserved on both active and backup paths.

The algorithm works as follows:

- STEP 1: Generate the logical topology for the network.

- STEP 2: Determine the active path (shortest path) for the requested call in the logical topology using the SLP algorithm

- STEP 3: Determine the shortest link-disjoint backup path for the requested call in the network using the SLP algorithm

- STEP 4: If both the active and the backup paths are available, the call is routed through the computed path, otherwise the requested call is blocked. 


\subsection{Complete shared protection}

In this case the information available is the set of active paths $A_{i j}$ that use link $(i, j)$ and the set of backup paths $B_{i j}$ that use link (i, j). The algorithm is same as the dedicated protection in section 2.1, except STEP 3 as follows:

- STEP 3: Compute the new set of costs for each link on the network. Let $\delta_{i j}{ }^{\mathrm{uv}}$ represent the total number of calls that use link $(i, j)$ for active path and link $(u, v)$ for backup path. A failure of any link used by the active path whose backup path is to be computed would lead to a capacity demand of not more than $\left(\delta_{i j}{ }^{u v}+1\right)$ on link $(u, v)$ that has to be used for the backup path of the active path under consideration. So, the cost for every link may be defined as [3]

$$
\begin{aligned}
& \phi_{\mathrm{ij}}^{\mathrm{uv}}=0, \quad \text { if } \delta_{\mathrm{ij}}^{\mathrm{uv}}+1 \leq \mathrm{G}_{\mathrm{uv}} \text { and }(\mathrm{i}, \mathrm{j}) \neq(\mathrm{u}, \mathrm{v}) \\
& =\left(\delta_{\mathrm{ij}}^{\mathrm{uv}}+1-\mathrm{G}_{\mathrm{uv}}\right) \text { if } \delta_{\mathrm{ij}}{ }^{\mathrm{uv}}+1>\mathrm{G}_{\mathrm{uv}} \text { and } \\
& R_{u v} \geq\left(\delta_{i j}{ }^{u v}+1-G_{u v}\right) \text { and }(i, j) \neq(u, v) \\
& =\infty, \quad \text { otherwise. }
\end{aligned}
$$

The task is to determine the shortest link disjoint backup path for the requested call in the network with new set of weights assigned to each link.

\subsection{Partial shared protection}

In this case for every link $(i, j)$ the aggregate bandwidth information $F_{i j}, G_{i j}$ and $R_{i j}$. are available. The information is independent of the number of calls that are currently present in the network. The algorithm for partial shared protection case is same as dedicated protection with only the suggested change in STEP 3 as follows:

- STEP 3: Compute the cost for each link on the network as

$$
\begin{aligned}
C_{u v} & =0, & & \text { if }(M+1) \leq G_{u v} \\
& =M+1-G_{u v} & & \text { if }(M+1)>G_{u v} \text { and } R_{u v}>\left(M+1-G_{u v}\right) \\
& =\infty & & \text { otherwise }
\end{aligned}
$$

where, $C_{u v}$ is the cost associated with link (u, v) in the backup path and $M$ representing the largest value of $F_{i j}$ for some link $(i, j)$ in the active path. Determine the shortest link-disjoint backup path for the requested call in the network with new set of weights assigned to each link. 


\subsection{Partial shared protection with exact reservation}

In the partial shared protection the link cost is based on a conservative value of M. A better performance of the algorithm can be achieved by exact reservation [3]. Let $M_{1}$ represent the active bandwidth in $F_{i j}$ in the active part for direct (s,d) pair calls only and $M_{2}$ represent the largest value of $F_{i j}$ excluding $M_{1}$ value in the active path whose backup path is going to be shared by current backup bandwidth and $M=M_{1}+M_{2}$. The algorithm for partial shared protection with exact reservation case is the same as the partial shared case except STEP 3 is changed as follows:

- STEP 3: Compute the cost for each link in the network as

$$
\begin{aligned}
C_{u v}=0, & \text { if } M+1 \leq G_{u v} \\
=M+B W-G_{u v} & \text { if } M+1>G_{u v} \text { and } \\
& R_{u v} \geq\left(M+1-G_{u v}\right) \\
=\infty, & \text { otherwise. }
\end{aligned}
$$

Determine the shortest link disjoint backup path for requested call in the network with new set of weights assigned to each link.

\section{PROTECTION TECHNIQUES IN INTEGRATED ROUTING}

In this section we consider the problem of integrated routing for restorable connections with and without backup sharing. The routing protocol makes use of the wavelength usage on physical links and bandwidth usage on IP logical links. We consider two protection strategies for integrated routing in IP over WDM networks viz. 1) dedicated path protection and 2) shared path protection.

\subsection{Dedicated path protection}

We consider the following definitions that are required for dedicated path protection strategy in integrated routing. Let

$\mathrm{R}_{\mathrm{uv}, \lambda} \rightarrow$ Residual capacity on $\lambda^{\text {th }}$ wavelength on link $(\mathrm{u}, \mathrm{v})$.

BW $\rightarrow$ Call request bandwidth.

$\mathrm{C}_{\mathrm{uv}, \lambda} \rightarrow$ Weight of $\lambda^{\text {th }}$ wavelength on link $(\mathfrak{u}, \mathrm{v})$. 
In this strategy, the information available to the path selection algorithm is only the residual capacities on each wavelength link. Hence, the complete resource required by the requested call has to be reserved on both active and backup paths. The algorithm works as follows:

- STEP 1: Construct the wavelength graph.

- STEP 2: Assign the weight to each wavelength link as given below.

$$
\begin{aligned}
\mathrm{C}_{\mathrm{uv}, \lambda}=1 \text { if } R_{u v, \lambda} \geq \mathrm{BW} \\
=\infty \text { otherwise. }
\end{aligned}
$$

- STEP 3: Compute the active path.

- STEP 4: Remove the fiber links through which the active path traverses.

- STEP 5: Assign the weight to each wavelength link as given below.

$$
\begin{aligned}
\mathrm{C}_{\mathrm{uv}, \lambda} & =1 \text { if } R_{u v, \lambda} \geq \mathrm{BW} \\
& =\infty \text { otherwise. }
\end{aligned}
$$

- STEP 6: Compute the backup path.

- STEP 7: If both active and backup paths are found, admit the call into the network. Otherwise block the call.

\subsection{Shared path protection}

We consider the following definitions that are required for shared path protection strategy in integrated routing. Let

$\mathrm{R}_{\mathrm{i}, \lambda} \rightarrow$ Residual capacity on $\lambda^{\text {th }}$ wavelength on link (i,j).

$F_{i j, \lambda} \rightarrow$ Active capacity on $\lambda^{\text {th }}$ wavelength on link $(i, j)$.

$\mathrm{G}_{\mathrm{ij}, \lambda} \rightarrow$ Backup capacity on $\lambda^{\text {th }}$ wavelength on link $(\mathrm{i}, \mathrm{j})$.

BW $\rightarrow$ Call request bandwidth.

$C_{i j, \lambda} \rightarrow$ Weight of $\lambda^{\text {th }}$ wavelength on link $(i, j)$.

$\phi_{\mathrm{ij}}{ }^{\mathrm{uv}, \lambda} \rightarrow \mathrm{F}_{\mathrm{ij}} \cap \mathrm{G}_{\mathrm{uv}, \lambda}=$ set of demands that use link (i,j) on active path and wavelength link $(\lambda, u, v)$ on the back up path.

$\delta_{\mathrm{ij}}^{\mathrm{uv}, \lambda} \rightarrow$ Sum of all demand values of set $\phi_{\mathrm{ij}}^{\mathrm{uv}, \lambda}$.

$\mathrm{M} \rightarrow$ Maximum value of $\delta_{i j}^{\mathrm{uv}, \lambda}$ for all $(\mathrm{i}, \mathrm{j})$ belonging to active path.

The algorithm given in section 4.1 is valid here also except STEP 5 .

- STEP 5: Assign the weight to each wavelength link as given below.

$$
\begin{array}{rlrl}
C_{u v, \lambda} & =0 & \text { if }(M+B W) \leq G_{u v, \lambda} \\
& =\left(M+B W-G_{u v, \lambda}\right) & \text { if }(M+B W)>G_{u v, \lambda} & \text { and } \\
& =\infty & & \left(M+B W-G_{u v, \lambda} \leq R_{u v, \lambda}\right)
\end{array}
$$

Applying the SLP algorithm to the weighted network graph, the edge disjoint backup path can be found out. 


\section{Simulation Results}

We simulate a dynamic network environment with the assumptions that the call requests process is Poisson and the call holding time follows a negative exponential distribution. Figure 1 shows a comparison of the blocking probability $P_{b}$ versus the total network load for a 14- node NSFNET utilizing 8 wavelengths for dedicated and partial shared protection cases. It may be noted that partial shared protection with exact reservation ensures the best blocking performance compared to the other schemes considered especially at lower loads.

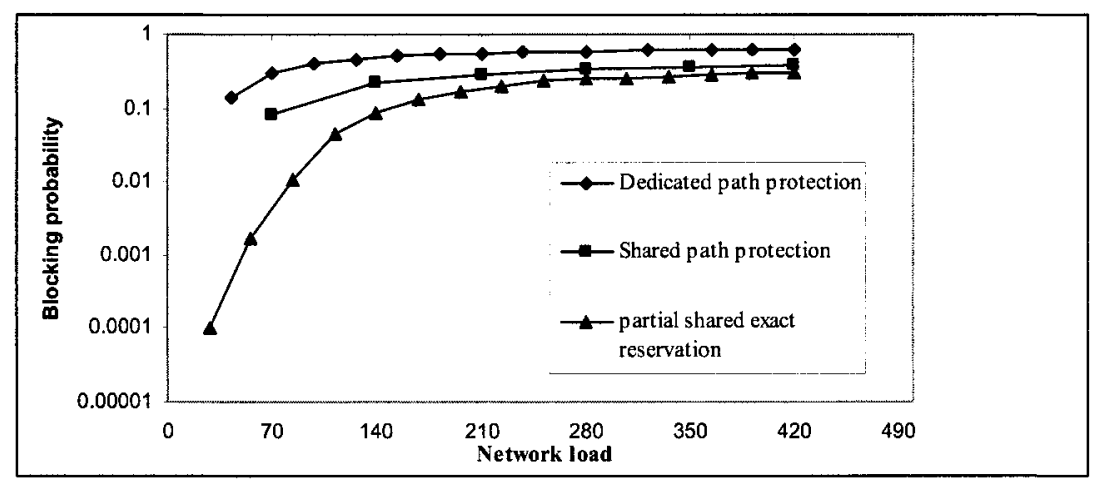

Figure. $1 . \mathrm{P}_{\mathrm{b}}$ vs. the network load for a 14 -node NSFNET, $\mathrm{W}=8$

Figure 2 shows the resource usage in terms of required number of wavelengths versus network load satisfying $P_{b} \leq 0.05$ for several protection strategies. The shared protection with exact reservation demands the minimum network resource.

In the case of integrated routing using the same NSFNET topology with 16 wavelengths, the results for the blocking probability and the network resource usage against network load for the dedicated protection as a function of $\mathrm{R}$ and BW are shown in Figs. 3 and 4 respectively. The performance is seen to improve significantly with increased number of router nodes and sub-lambda traffic grooming. Similar trend is also noticed in the results for the integrated shared path protection case with much improved performance compared to those in dedicared protection case as depicted in Fig.5 


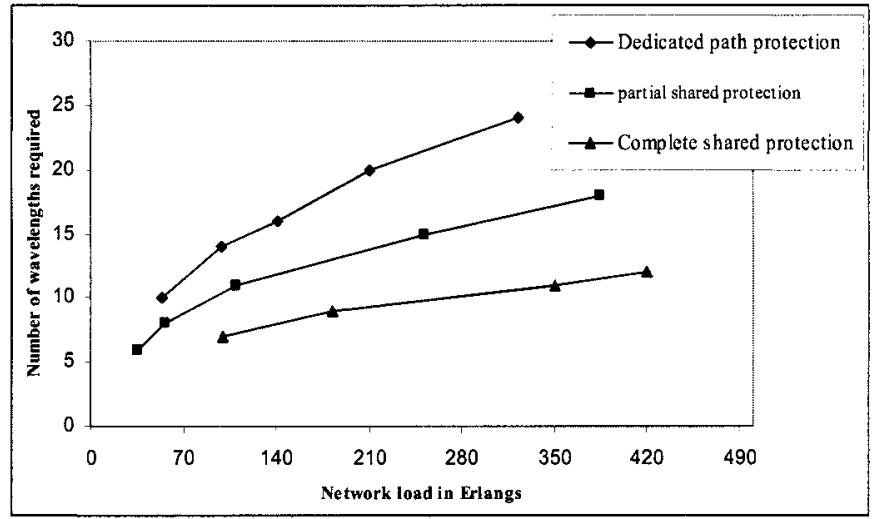

Figure 2. Resource usage versus network load, for $\underline{P}_{\mathrm{b}} \leq 0.05$ for several protection strategies

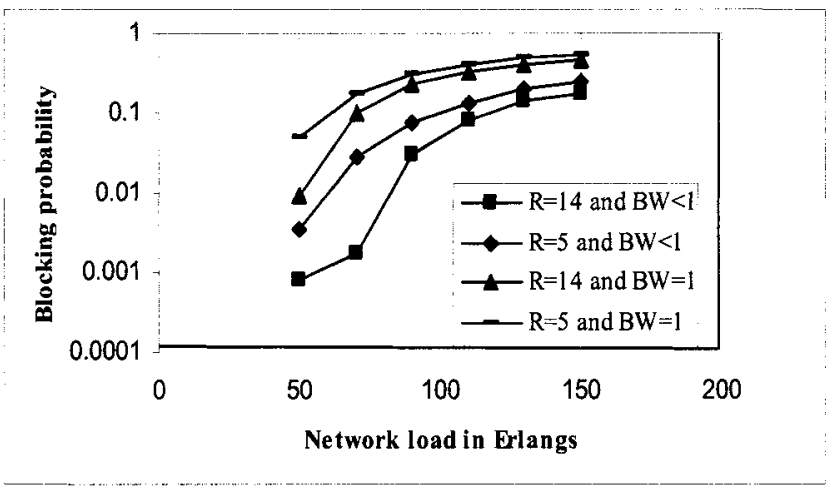

Figure 3. $\mathrm{P}_{\mathrm{b}}$ versus network load for a 14-node NSFNET, dedicated path protection, $\mathrm{R} \equiv$ number of router nodes, $\mathrm{BW} \equiv$ call bandwidth, $\mathrm{W}=16$

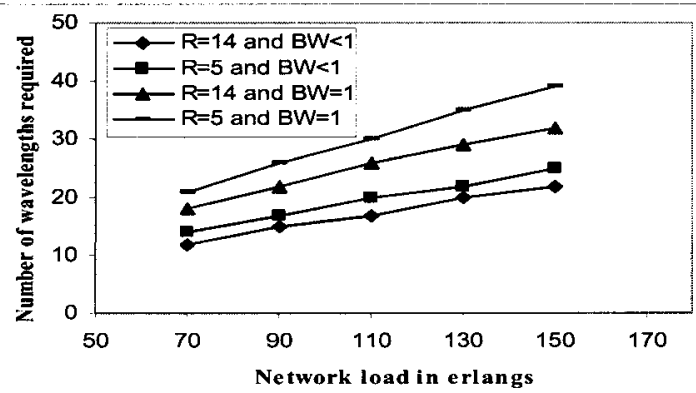

Figure 4. Number of wavelengths required versus network load for a 14node NSFNET dedicated path protection in integrated routing. 


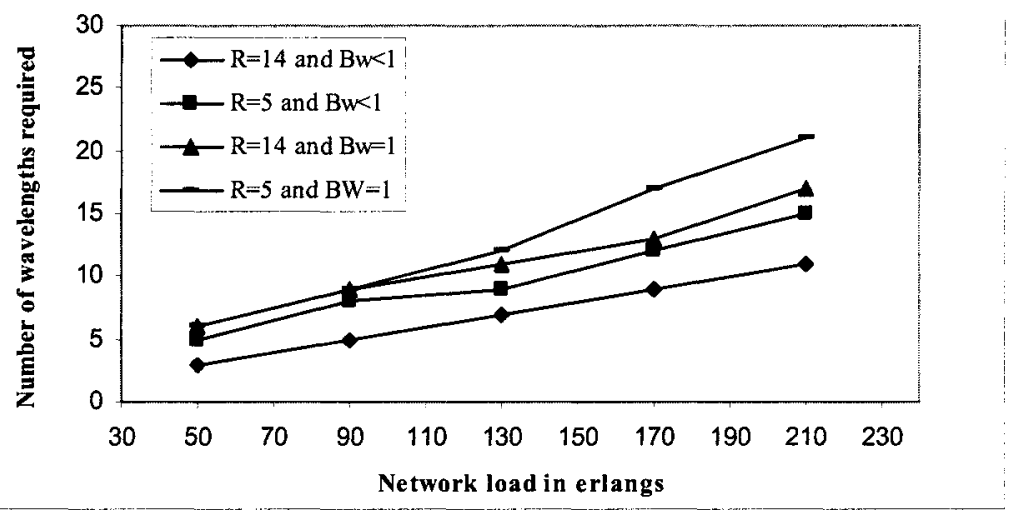

Figure 5 Number of wavelengths required versus network load for a 14node NSFNET shared path protection in integrated routing.

\section{CONCLUSION}

Based on SLP algorithm in finding both active and backup paths, several bandwidth guaranteed protection strategies depending on the link state information available have been applied in WDM mesh network. The simulation results indicate that partial shared protection with exact reservation achieves the best network blocking probability performance and resource requirement. In the integrated routing scenario in IP-over-WDM network shared path protection achieves significant performance gain if the network contains more number of router nodes than pure OXC nodes and facilitates more sub-lamda granurality traffic multiplexing.

\section{REFERENCES}

[1]. M. Kodialam and T.V. Lakshman, "Restorable Dynamic Quality of Service Routing", IEEE Communication Magazine, June 2002, pp 72-81.

[2]. M. Kodialam and T.V. Lakshman, "Dynamic Routing of Bandwidth Guaranteed paths with Restoration", Proc. Infocom, 2002.

[3]. I.Chlamtac, A.Farago and T. Zhang "Lightpath (wavelength) Routing in Large WDM Networks" IEEE Journal on selected areas in communications, June 1996, vol. 14, no.5 pp: 909-913. 